\title{
A Hybrid TD-AD Algorithm Based Electromagnetic Spectrum Assignment Method
}

\author{
L.Guo \\ PLA University of Science and Technology \\ Nanjing, China
}

\author{
T. Liang \\ Nanjing Telecommunication Technology Institute \\ Nanjing, China
}

\begin{abstract}
The object of spectrum assignment is to build its own ordered spectrum environment and maintain uninterruptedly spectrum resource control. In this article, we build a mathematical model and put forward the hybrid TD (table dispatching method) and the AD (auxiliary decision method) algorithm. The results show that this method is contributed to minimize the allocation time, reduce the technical background demand and improve the decision veracity.
\end{abstract}

Keywords-spectrum assignment; auxiliary decision; optimization allocation

\section{INTRODUCTION}

It is a paradox in the area of wireless communication that total frequency spectrum available has been exhaust while much of them have been fairly underutilized [1]. But to the best of our knowledge, there exist only a few literatures - which focused on spectrum decision, among which few take both of short-term and long-term information into consideration. Nowadays, due to the booming informationized weapon industry and the comprehensive strengthen of equipment informationization, the spectrum environment of vessel formation is more and more complicate. To solve the problem of artificial spectrum shortage and deployable difficulty, we analysis two aspect problems: build the mathematical model and combine the table dispatching method and the auxiliary decision to solve the allocation question [2].

\section{The SPECTRUm Allocation ProJect Design}

Due to the development of the mode of operations, it is the common view that frequency is as important as bullet. We characterize the mathematical model as follows: (1) The vessel formation scenes are divided into several different scene; (2) We analysis the interference pattern and spectrum management demand of specific scene, then we get the optimal spectrum management program and put the scene and spectrum management program to the CBR (Case-Based Reasoning) library ;(3) The spectrum allocation question of the vessel formation will be seen as a new scene. If there is no same scene, we will use the suggested arithmetic to get the solution.

The spectrum allocation mathematical model is characterized as follows: how to find the best spectrum allocation matrix $A$ to let the benefit $U(R)$ be maximum for the

\author{
A.W. Sun, Y.J. Zhang \\ PLA University of Science and Technology \\ Nanjing, China
}

spectrum matrix $L$, benefit matrix $B$, spectrum interference matrix $C$ have be known [3].

Suppose there is $N$ equipment and $M$ frequency band. We define the spectrum matrix $L$, benefit matrix $B$, spectrum interference matrix $L$, best spectrum allocation matrix $A$ and the benefit $U(R)$ :

Available spectrum matrix $L$ :

$$
L=\left\{l_{m, n} \mid l_{m, n} \in\{0,1\}\right\}_{N \times M}
$$

$\mathrm{l}_{\mathrm{m}, \mathrm{n}}=1$ denotes the equipment $\mathrm{n}(1 \leq \mathrm{n} \leq \mathrm{N})$ can use spectrum $\mathrm{m}(1 \leq \mathrm{m} \leq \mathrm{M})$

Benefit matrix B:

$$
\begin{aligned}
& \quad B=\left\{b_{m, n}\right\}_{N \times M} \\
& \mathrm{~B}=\left\{\mathrm{b}_{\mathrm{m}, \mathrm{n}}\right\}_{\mathrm{N} \times \mathrm{M}} \text { is the benefit when equipment } \mathrm{n} \\
& (1 \leq \mathrm{n} \leq \mathrm{N}) \text { use spectrum } \mathrm{m}(1 \leq \mathrm{m} \leq \mathrm{M}) \text {, of cause, } \mathrm{b}_{\mathrm{m}, \mathrm{n}}=0 \\
& \text { when } \mathrm{l}_{\mathrm{m}, \mathrm{n}}=0 \text {. }
\end{aligned}
$$

Spectrum interference matrix $\mathrm{C}$ :

$$
C=\left\{c_{n, k, m} \mid c_{n, k, m} \in\{0,1\}\right\}_{N \times N \times M}
$$

$\mathrm{C}_{\mathrm{n}, \mathrm{k}, \mathrm{m}}=1$ shows interference exist when equipment $\mathrm{n}$ and $\mathrm{k}(1 \leq \mathrm{n}, \mathrm{k} \leq \mathrm{n})$ use spectrum $\mathrm{m}(1 \leq \mathrm{m} \leq \mathrm{M})$ at the same time.

No interference matrix A:

$$
A=\left\{a_{n, m} \mid a_{n, m} \in\{0,1\}\right\}_{N \times M}
$$

$\mathrm{a}_{\mathrm{n}, \mathrm{m}}=1$ shows the equipment $\mathrm{n}(1 \leq \mathrm{n} \leq \mathrm{N})$ use spectrum $\mathrm{m}(1 \leq \mathrm{m} \leq \mathrm{M})$.

The whole benefit can describe by:

$$
R=\left\{r_{n}=\sum_{m=1}^{M} a_{n, m} \times b_{m, n}\right\} .
$$

So we can easily get that the spectrum allocation question can transform into the following optimization problem:

$$
A^{*}=\arg \max U(R)
$$




\section{THE MATHEMATICAL MODEL}

\section{A. Table Dispatching Method to Solve Spectrum Allocation Question}

In the case of interference, the objective of spectrum allocation of vessel formation is to guarantee that the equipments which have priority can use the spectrum by the means of reasonable spectrum allocation [4]. It is contain two problems: first, get the priority of this equipment; second, confirm the spectrum allocation as effective as possible.

Suppose there is $N$ equipment and $\mathrm{M}$ frequency band. The priority weight of the equipment in different spectrum band can be described as the following matrix:

$$
\omega=\left[\begin{array}{cccc}
\omega_{11} & \omega_{12} & \cdots & \omega_{1 N} \\
\omega_{21} & \omega_{22} & \cdots & \omega_{2 N} \\
\cdots & \cdots & \cdots & \cdots \\
\omega_{M 1} & \omega_{M 2} & \cdots & \omega_{M N}
\end{array}\right]
$$

where $\omega_{\mathrm{ij}}$ is the priority weight of $\mathrm{j}$ equipment when it use ith spectrum band.

We translate the priority weight matrix $\omega$ into cost matrix Z. $Z_{i j}=1-\omega_{i j}$.

$$
Z=\left[\begin{array}{cccc}
z_{11} & z_{12} & \cdots & z_{1 N} \\
z_{21} & z_{22} & \ldots & z_{2 N} \\
\cdots & \cdots & \ldots & \ldots \\
z_{M 1} & z_{M 2} & \cdots & z_{M N}
\end{array}\right]
$$

For example, suppose there is five equipment and three frequency band. The priority weight matrix is:

$$
\omega=\left[\begin{array}{ccccc}
0.4 & 0.45 & 0.3 & 0.4 & 0.2 \\
0.2 & 0.35 & 0.45 & 0.25 & 0.3 \\
0.4 & 0.2 & 0.25 & 0.35 & 0.5
\end{array}\right]
$$

Then the cost matrix is:

$$
Z=\left[\begin{array}{ccccc}
0.6 & 0.55 & 0.7 & 0.6 & 0.8 \\
0.8 & 0.65 & 0.55 & 0.75 & 0.7 \\
0.6 & 0.8 & 0.75 & 0.65 & 0.5
\end{array}\right]
$$

Suppose the free frequency band are $2 \sim 18 \mathrm{MHz}$, $20 \mathrm{MHz} \sim 27 \mathrm{MHz}, 31 \mathrm{MHz} \sim 45 \mathrm{MHz}$.And the frequency band needed is $6 \mathrm{MHz}, 7 \mathrm{MHz}, 10 \mathrm{MHz}, 6 \mathrm{MHz}, 8 \mathrm{MHz}$.

TABLE I. MATRIX DATE

\begin{tabular}{l|l|l|l|l|l|ll}
\hline$A_{i}$ & $B_{1}$ & $B_{2}$ & $B_{3}$ & $B_{4}$ & $B_{5}$ & $a_{i}$ \\
$B_{j}$ & & & & & & & \\
\hline $\mathrm{A}_{1}$ & 0.6 & 0.55 & 0.7 & 0.6 & 0.8 & 16 & \\
\hline $\mathrm{A}_{2}$ & 0.8 & 0.65 & 0.55 & 0.75 & 0.7 & 7 & \\
\hline $\mathrm{A}_{3}$ & 0.6 & 0.8 & 0.75 & 0.65 & 0.5 & & 14 \\
\hline $\mathrm{b}_{\mathrm{j}}$ & 6 & 7 & 10 & 6 & 8 & \\
\hline \hline
\end{tabular}

where $\overline{A_{i}}$ is the $i$ th spectrum, and $a_{i}$ is the frequency band of $A_{i}, B_{j}$ is the $j$ th equipment, and $b_{j}$ is the frequency band of $B_{j}$ needed. Every check corresponds to $z_{i j}$. First, we use northwest corner method to get, the initial solution. The calculate result

is $\left(x_{11} x_{12} x_{13} x_{23} x_{33} x_{34} x_{35}\right)=(6,7,3,7,0,6,8)$. The objective function value is $f=21.3$. Set the initial solution into table 2 . Calculate the $\omega_{i}$ and $v_{j}$, set them into the left column and the first line. Then calculate $z_{i j}-c_{i j}$. The reduced cost of basic variable is 0 .

TABLE II. MAX REDUCED

\begin{tabular}{l|l|l|l|l|l|l|l}
\hline \hline & $v_{j}$ & 0.6 & 0.55 & 0.7 & 0.6 & 0.45 & \\
\hline$\omega_{i}$ & $A_{i}$ & $B_{1}$ & $B_{2}$ & $B_{3}$ & $B_{4}$ & $B_{5}$ & $a_{i}$ \\
\hline 0 & $A_{1}$ & 0 & 0 & 0 & 0 & -0.35 & 16 \\
\hline-0.15 & $A_{2}$ & -0.35 & -0.25 & 0 & -0.3 & -0.4 & 7 \\
\hline 0.05 & $A_{3}$ & 0.05 & -0.2 & 0 & 0 & 0 & 14 \\
\hline & $b_{j}$ & 6 & 7 & 10 & 6 & 8 & \\
\hline \hline
\end{tabular}

On table 2 , the max reduced cost is $z_{31}-c_{31}=0.05$. It does not reach the optimal result .Then we use the closed-lope method to improve the initial solution. Take the closed-lope $x_{11} x_{31} x_{14} x_{34}$, let the adjustment amount be $\theta$. To maintain the feasibility, let $x_{31}=\theta \geq 0, x_{34}=6-\theta \geq 0, x_{11}=6-\theta \geq$ $0, x_{14}=\theta \geq 0$, get $\theta=6$. The new basic variable is $\left(x_{12} x_{13} x_{14} x_{23} x_{31} x_{33} x_{35}\right)=(7,3,6,7,6,0,8)$. The objective function value is $f=21$. Then calculate $z_{i j}-c_{i j}$. The result shows that whole reduced costs are negative or zero. So we have get the best result as follws: ${ }_{B_{1}}: 2 \mathrm{MHz} \sim 8 \mathrm{MHz}, B_{2}$ : $8 \mathrm{MHz} \sim 15 \mathrm{MHz} \quad, \quad B_{3}: 15 \mathrm{MHz} \sim 18 \mathrm{MHz}, B_{4}:$ $31 \mathrm{MHz} \sim 37 \mathrm{MHz}, \quad B_{5}: 37 \mathrm{MHz} \sim 45 \mathrm{MHz} \cdot$

\section{B. Similarity Retrieval Algorithm Model Based on CBR}

The similarity retrieval flow based on CBR is described by picture2:

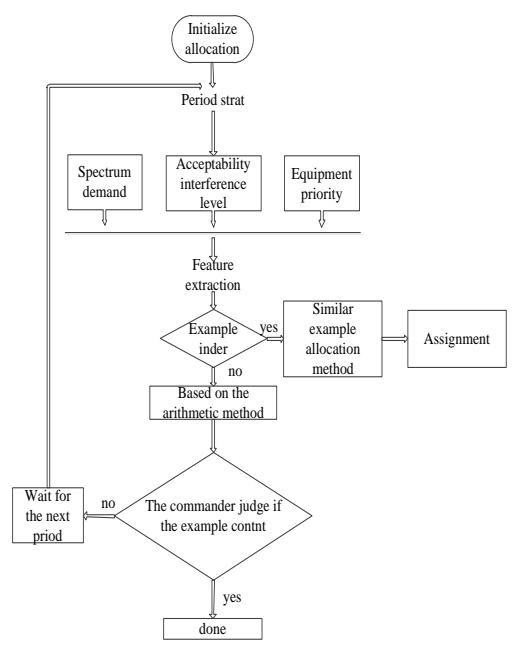

FIGURE I. SIMILARITY RETRIEVAL FLOW BASED ON CBR 
The process is: let the spectrum assignment be new example, and get the feature extraction. Then we check the CBR library. If there is similar scene, we get the auxiliary decision to report to the commander; otherwise, use the suggested arithmetic to get the solution [5].First, we calculate the relative distance between new scene and the example of CBR library

$$
d\left(V_{r}^{\prime}, V_{r}^{i}\right)=\left|\frac{V_{r}^{\prime}-V_{r}^{i}}{V_{r}^{\prime}}\right|
$$

where $V_{r}{ }^{\prime}$ and $V_{r}{ }^{i}$ are $r$ th characteristic value of ${ }_{i}$ th example. The characteristic value of this paper is $z_{i j}$. Then calculate the similarity between $V^{\prime}$ and $V^{i}$

$$
S D\left(V^{\prime}, V^{i}\right)=1-\sum_{r=1}^{n} \omega_{r} \cdot d\left(V^{\prime}, V^{i}\right)
$$

The new scene and the example of CBR library are similar when the similarity Approach 1[6].We can set the threshold [7].(For example, set the threshold be 0.9). When the similarity between $\mathrm{V}^{\prime}$ and $V^{i}$ bigger than 0.9 , we can get the auxiliary decision to report to the commander[8].

\section{CONCLUSION}

In this paper, we analysis the spectrum allocation method. A mathematical model used in spectrum assignment is developed. We build the mathematical model and combine the table dispatching method and the auxiliary decision to solve the allocation question. The results show the performance improvements of the proposed algorithms. So it has very important practical significance.

\section{ACKNOWLEDGEMENTS}

This research is supported in part by the Social Sciences Foundation of China under Grant 13GJ003-070 and the Nature Science Foundation of China under Grant 61471392.

\section{REFERENCES}

[1] STAFF J. Electronic warfare [M]. Washington: Joint Staff, 2007.

[2] STAFF J. Joint operations in the electromagnetic battle space[M]. Washington: Joint Staff, 2000.

[3] D. Feng, C. Jiang, G. Lim, L. Cimini, G. Feng, and G. Li, A survey of energy-efficient wireless communications, IEEE Commun. Surv. \&Tutor., vol. 15, no. 1, pp. 167-178, 2013.

[4] X. Gong, G. Dartmann, A. Ispas, G. Ascheid. Optimal power allocation in a spectrum sharing system with partial CSI.in Proc. IEEE Veh. Tech. Conf. - Spring, pp. 1-5. 2012.

[5] M. Khoshnevisan, J. Laneman. Power allocation in multi-antenna wireless systems subject to simultaneous power constraints.IEEE Trans. Commun., vol. 60, no. 12, pp. 3855-3864, Dec. 2012.

[6] Won-Yeol Lee, Ian F. Akyildiz. A Spectrum Decision Framework for Cognitive Radio Networks.IEEE Transactions on Mobile Computing, vol. 10, no. 2, pp.161-174, 2011.

[7] Y. Xie, B. Armbrustery, Y. Ye,Dynamic Spectrum Management with the Competitive Market Mode, IEEE Transactions on SIGNAL Processing, Vol. 58, No. 4, April 2010.

[8] B.Wang, K. Liu, J.Ray. Advances in Cognitive Radio Networks: A Survey .IEEE Journal of Selected topics in signal processing, vol. 5., no. 1, pp. 5-23, 2011. 